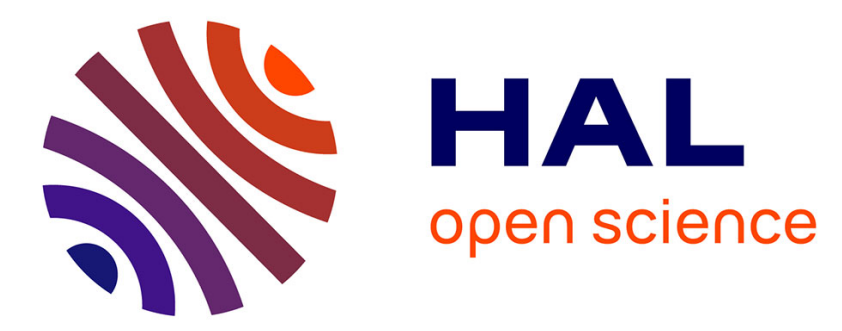

\title{
Framework for Hierarchical and Distributed Smart Grid Management
}

\author{
Rémi Bonnefoi, Christophe Moy, Jacques Palicot
}

\section{To cite this version:}

Rémi Bonnefoi, Christophe Moy, Jacques Palicot. Framework for Hierarchical and Distributed Smart Grid Management. XXXIInd International Union of Radio Science General Assembly \& Scientific Symposium (URSI GASS), URSI, Aug 2017, Montreal, Canada. 10.23919/ursigass.2017.8104500 . hal-01504251

\section{HAL Id: hal-01504251 \\ https://hal.science/hal-01504251}

Submitted on 9 Apr 2017

HAL is a multi-disciplinary open access archive for the deposit and dissemination of scientific research documents, whether they are published or not. The documents may come from teaching and research institutions in France or abroad, or from public or private research centers.
L'archive ouverte pluridisciplinaire HAL, est destinée au dépôt et à la diffusion de documents scientifiques de niveau recherche, publiés ou non, émanant des établissements d'enseignement et de recherche français ou étrangers, des laboratoires publics ou privés. 


\title{
Framework for Hierarchical and Distributed Smart Grid Management
}

\author{
Rémi Bonnefoi $^{(1)}$, Christophe Moy ${ }^{(1)}$, and Jacques Palicot ${ }^{(1)}$ \\ (1) CentraleSupélec/IETR, CentraleSupélec Campus de Rennes, 35510 Cesson-Sévigné, France
}

\begin{abstract}
We use the Hierarchical and Distributed Cognitive Radio Architecture Management (HDCRAM) inherited from cognitive radio as a generic framework for smart grid management. This architecture is an efficient tool which can be used for the design of decision making algorithms in complex systems. We apply this architecture on the smart grid and we identify both intelligent entities and communication networks which compose the management part of the smart grid. Furthermore, we consider which radio standards can be used for each link.
\end{abstract}

\section{Introduction}

In a smart grid, information and communication technologies are used to improve reliability, security and efficiency of the electrical network [1]. On the one hand, decision making algorithms are used to manage the grid. On the other hand, a communication system is necessary to transfer the information between the entities which compose the smart grid, e.g. sensors and decision making (intelligent) entites. Furthermore, in a smart grid, the management entities are heterogeneous (in term of processing capabilities, energy consumption, etc.), distributed and used for various applications. In such scenario, efficient management strategies have to be built on a framework. That is why several hierarchical architecture have been proposed as frameworks for smart grid management. In [2], the authors propose a hierarchical architecture for microgrid management. In [3], a three level architecture is proposed for the management of the Advanced Metering Infrastrucutre (AMI). However, these architectures are designed for only one application or one part of the smart grid. The Smart Grid Coordination Group (SG-CG) has proposed the Smart Grid Architecture Model (SGAM) as a general framework for smart grid management $[4,5]$. The SGAM architecture focuses on the interoperability between the different part of the smart grid. In [6], the authors of this paper proposed the Hierarchical and Distributed Cognitive Radio Architecture Management (HDCRAM) for the management of the smart grid. However, in our previous work, we did not consider that the different parts of the grid are managed by different mechanisms and can be managed independently. In this paper we show that the HDCRAM architecture can be used for the management of the different parts of the grid and we also identify the communication networks that should be used appropriately at each level of management.

The aim of this paper is to describe the HDCRAM architecture as a generic framework for smart grid management. We show that HDCRAM can be used either for the management of the electricity production and consumption or for transmission and distribution grids automation. We identify the management entities and the communication networks used between these entities.

The rest of this paper is organized as follows. The communication networks used for the smart grid are described in section 2. HDCRAM architecture is described in section 3. In section 4, we use HDCRAM as a framework for smart grids and we identify the role of the intelligent entities and of the communication networks in the architecture. Finally, section 5 concludes this paper.

\section{Communication Networks for the Smart Grid}

Several communication networks must be used for the management of the smart power grid. In [4], the Smart Grid Coordination Group describes fourteen communication networks used for smart grid management. In table 1, we have sorted the networks which could be used for smart grid management by range: short range (e.g. Home Area Network (HAN), Neighbourhood Area Network (NAN) and Wide Area Network (WAN)) and depending on the application field. We consider three application fields; consumer side, production side and grid monitoring. The main networks used for the management of the power consumption are used for home automation and for the advanced Metering Infrastructure (AMI). The production field includes all the networks used for the management of power plants (nuclear plants, solar plants, etc.). The last field includes all the communication networks used for the distribution and transmission grids automation which is mainly done in substations. Smart grid communications are done through many networks which will use several communication technologies. In such situation with multi-standard communications, it is interesting to use a cognitive radio approach based on software defined radio (SDR) [7].

All the networks described in table 1 have their own constraints in term of throughput, latency, reliability and security [8]. As a consequence, the communication technolo- 


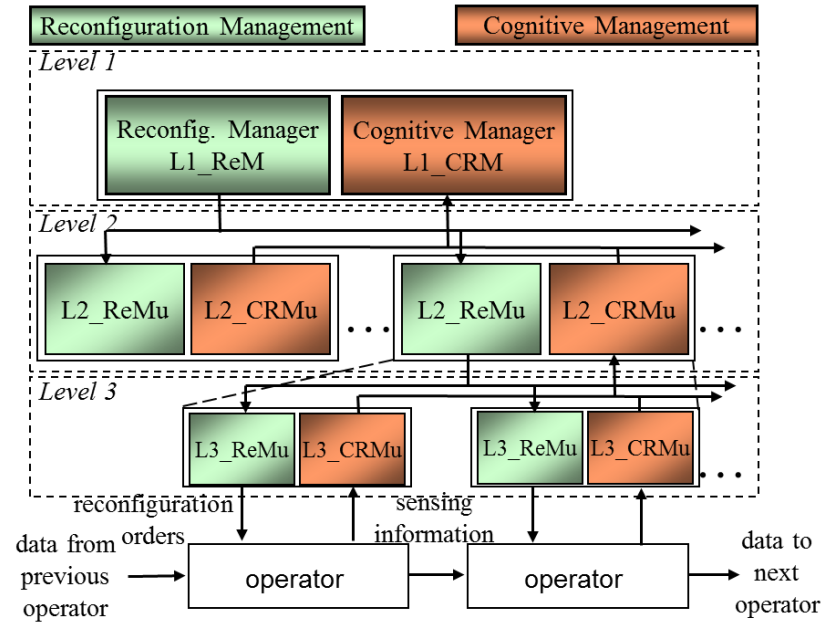

Figure 1. HDCRAM has 3 management levels and is composed by two independant paths. A cognitive path from bottom to top for retrieving sensing information to cognitive units and a configuration path from top to bottom.

gies have to be adapted to the network requirement. We have listed in table 1 some of the wireless standards which can be used for each network. A more complete list can be found in [5].

\section{Hierarchical and Distributed Cognitive Radio Architecture Management}

The Hierarchical and Distributed Cognitive Radio Architecture Management (HDCRAM) is a logical architecture which was first proposed for the management of cognitive radio systems [9]. This three levels architecture is shown in Figure 1.

This architecture comprises two managing sub-parts. The first part is the cognitive part featuring Cognitive Radio Management Units (CRMu): a CRMu exchanges information from the lower level to the upper level only. This entity has intelligent capabilities, and can make decisions and alert the upper-level CRMu. The second part is the reconfiguration part featuring Reconfiguration Management Units (ReMu): a ReMu exchanges information from the upper to the lower level only. This entity receives reconfiguration orders from the upper level and executes or transmits them to the lower level ReMu. Moreover, this architecture has three levels of decision. The first level is composed of the couple L1_CRM/L1_ReM which is unique and is the general manager of the system. The level L2 is an intermediate level composed by several couples L2_CRMu/L2_ReMu. L2 acts as a compression level in order to reduce the information sent to level 1. Finally, level 3 is composed of several couples (L3_CRMu/L3_ReMu). Each of these couples is associated to an operator of the power grid and each operator is associated to a L3 unit. These units are the link between the management part of the architecture and the smart grid operators. The decision can be made at any level. Local decisions are made at level 3. Level 2 is used to make decisions which concern several operators whereas level 1 is used for global decisions.

\section{Communication Networks in HDCRAM for Smart Grid}

\subsection{HDCRAM for Grid Production and Con- sumption Management}

One of the main tasks of smart grid management is to balance the electricity production and consumption to avoid the deterioration of the grid. Consequently, the proposed management architecture must handle both production and consumption part of the smart grid.

The management of the production and consumption in a smart grid can be viewed at different levels. In figure 2, HDCRAM architecture is used for the local management of the grid. The proposed three level architecture shows the management from smart appliances to the local substation. Level 1 manager is in the substation and can make the link between the local grid (which can be a microgrid) and the rest of the distribution network.

In figure 3, the management architecture is used at a higher level. Smart homes, buildings, power plants and other energy producers and consumers are connected to the level 3 entities and the level 1 entity is a global manager of the production and consumption. This entity manages the production and consumption over a large area (e.g. a country).

Both proposed HDCRAM architectures of figure 2 and 3 show the different communication networks which are used between the management entities. Most of the networks used for the management of the production and consumption part of the grid do not have tight constraints in reliability (packet delivery ratio) and latency. For each of these networks, a large number of communication technologies can be used as given in table 1. As an example, Wifi, Bluetooth or Zigbee can be used for local networks and cellular or Low Power Wide Area Networks can be used for long range communications.

\subsection{HDCRAM for Transmission Grid Man- agement}

The management of the electricity production and consumption is not the only mechanism used for the management of the grid. Indeed, sensors and actuators are also used for distribution and transmission grids automation. The management of the transmission part of the grid is done by Phasor Measurement Units (PMUs) and Intelligent Electronic Devices (IEDs) [10]. The information collected by PMUs is transmitted to the Phasor Data Concentrator (PDC) which can transmit it to the transmission coordinator. HDCRAM architecture used for the management of the transmission grid is shown in figure 4. 
Table 1. Communication networks for different levels of smart grid management

\begin{tabular}{|l|l|l|l|}
\hline Field & Range & networks & Wireless Technologies and standards \\
\hline \multirow{4}{*}{ Consumer field } & $\begin{array}{l}\text { Short range } \\
\text { (HAN) }\end{array}$ & $\begin{array}{l}\text { Subscriber access network, Home automa- } \\
\text { tion }\end{array}$ & Zigbee, Bluetooth, Wifi \\
\cline { 2 - 4 } & NAN & $\begin{array}{l}\text { Neighborhood access network, Backbone } \\
\text { network }\end{array}$ & Zigbee, Wifi, WirelessHART \\
\cline { 2 - 4 } & WAN & AMI backhaul & $\begin{array}{l}\text { GSM, GPRS, UMTS, LTE, WiMAX, Lo- } \\
\text { RaWAN, NB-IoT, Sigfox }\end{array}$ \\
\hline \multirow{2}{*}{ Grid monitoring } & Short range & Intra-substation Low-end intra-substation & $\begin{array}{l}\text { Most of the protocols envisioned for this } \\
\text { network are wired, e.g. IEC 61850 }\end{array}$ \\
\cline { 2 - 4 } & WAN & Inter-substation & LTE, LTE-M, NB-IoT, 5G \\
\hline \multirow{2}{*}{ Production field } & Short range & Industrial fieldbus & $\begin{array}{l}\text { GSM, GPRS, UMTS, LTE, WiMAX, Lo- } \\
\text { RaWAN, NB-IoT, Sigfox }\end{array}$ \\
\cline { 2 - 4 } & WAN & Balancing network & \\
\hline
\end{tabular}

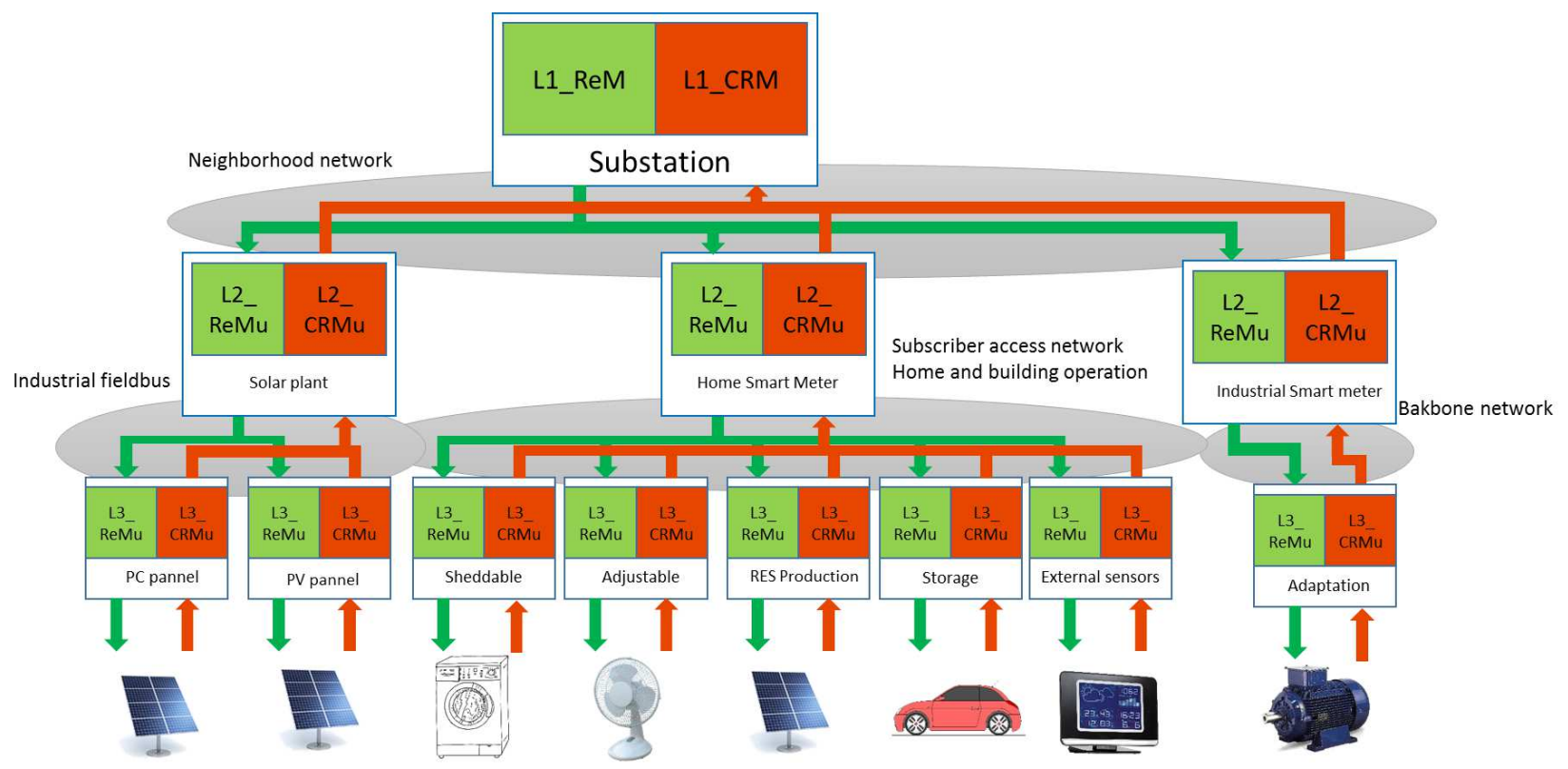

Figure 2. The HDCRAM architecture used as a management architecture for the local management of the electricity consumption.

Only one communication network is used for the transmission grid management. The inter-substation network is a wide area network with stringent constraints in latency and reliability [5]. In [11], it is specified that the communications latency for some PMUs applications (e.g. islanding) should not exceed $50 \mathrm{~ms}$. Consequently, only a few technologies can be used. Most of these technologies are wired technologies but the LTE standard can meet these communication requirements as given in table 1 .

\subsection{HDCRAM for Distribution Grid Man- agement}

Compared to transmission grid, the distribution grid is currently being considerably improved to handle renewable energy resources. Mechanisms similar to those used for the transmission grid will be used for the distribution grid. The management of the smart distribution grid is done through smart sensors and actuators. The framework used for distribution grid management is shown in figure 5.

In [5], the inter-substation network is used for both the transmission and distribution part of the grid. However, with the integration of renewable energy resources and the development of microgrids, the distribution grid will require more stringent constraints than the transmission grid. In [12], a $4 \mathrm{~ms}$ latency is required for distribution grid monitoring. This stringent requirements can be met with wired or wireless protocols specifically designed for ultra lowlatency communications. 


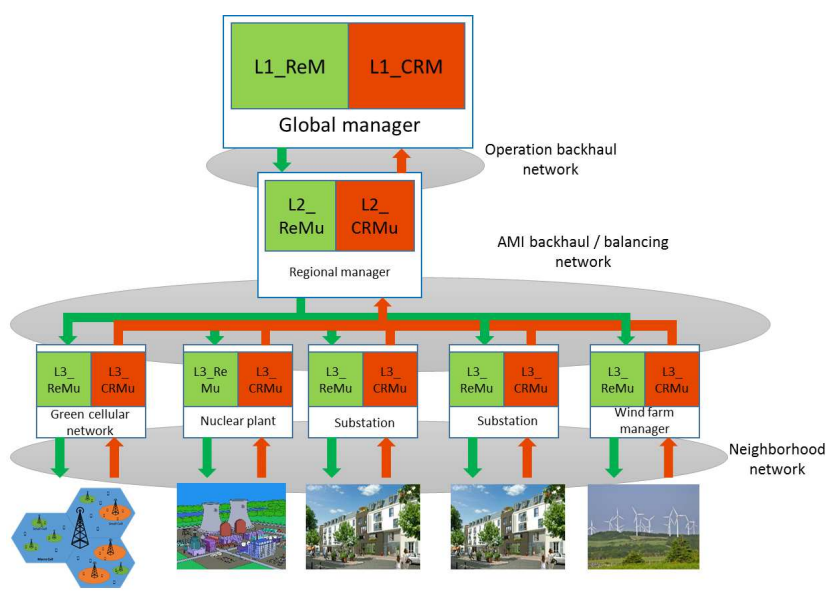

Figure 3. The HDCRAM architecture used as a management architecture for the global management of the electricity production and consumption

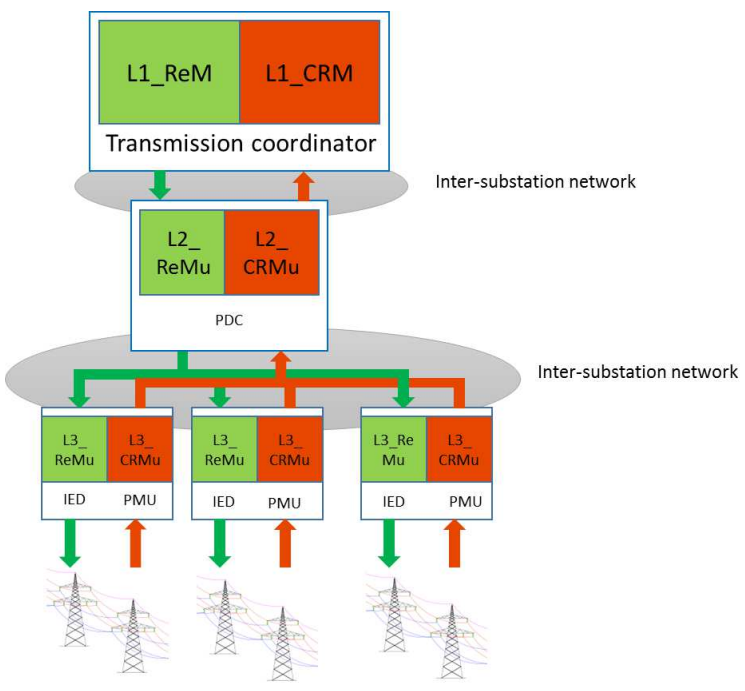

Figure 4. HDCRAM architecture used for the management of the transmission grid.

\section{Conclusion}

In this paper, the HDCRAM architecture is presented as a general framework for smart grid management. We show that many networks have to be used in a smart grid. Each network has specific constraints which depends on the application and on the range. We have mapped HDCRAM architecture on the different parts of the grid and we have identified the different management entities and the networks which compose the future grid. These architectures can represent the same part of the grid with a different granularity. This can ease the interconnection between the different visions.

\section{Acknowledgements}

Part of this work is supported by the project SOGREEN (Smart pOwer GRid for Energy Efficient small cell Networks), which is funded by the French national research agency, under the grant

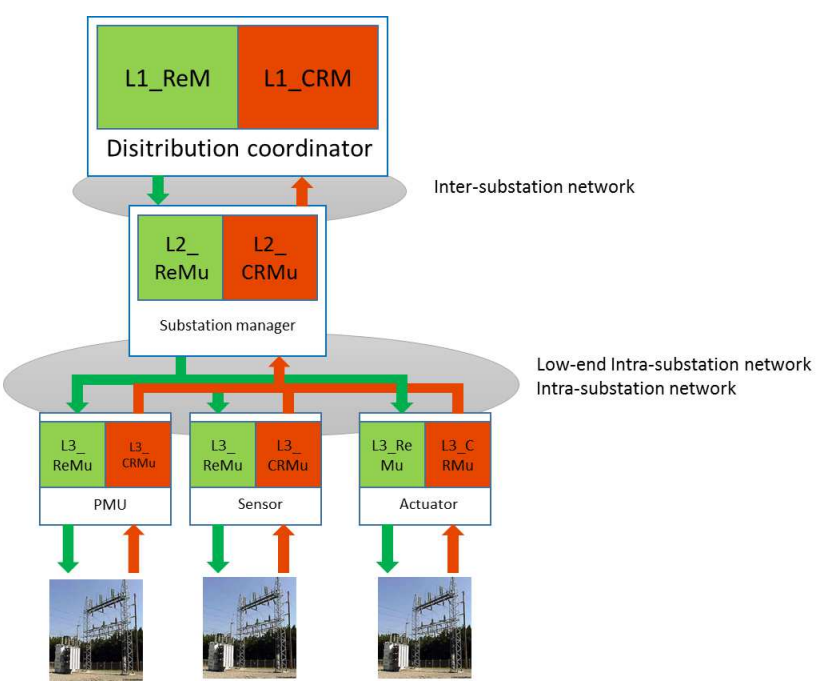

Figure 5. HDCRAM architecture used for the management of the distribution grid.

agreement coded: N ANR-14-CE28-0025-02 and by Région Bretagne, France.

\section{References}

[1] K. Moslehi and R. Kumar. A reliability perspective of the smart grid. IEEE Transactions on Smart Grid, 1(1):57-64, June 2010.

[2] C. Dou and B. Liu. Multi-agent based hierarchical hybrid control for smart microgrid. Smart Grid, IEEE Transactions on, 4(2):771-778, June 2013.

[3] J. Lloret, J. Tomas, A. Canovas, and L. Parra. An integrated iot architecture for smart metering. IEEE Communications Magazine, 54(12):50-57, December 2016.

[4] CEN-CENELEC-ETSI Smart Grid Coordination Group. Overview of SG-CG Methodologies, August 2014.

[5] CEN-CENELEC-ETSI Smart Grid Coordination Group. Smart Grid Reference Architecture, November 2012.

[6] J. Palicot and al. Application of hierarchical and distributed cognitive architecture management for the smart grid. Ad Hoc Networks, 41:86 - 98, 2016.

[7] J. Palicot. Radio Engineering: From Software Radio to Cognitive Radio. Wiley-ISTE, August 2011.

[8] Department of Energy of USA. Communications Requirements of Smart Grid Technologies, October 2010.

[9] L. Godard, C. Moy, and J. Palicot. An executable metamodel of a hierarchical and distributed architecture management for cognitive radio equipments. annals of telecommunications, 64(7-8):463-482, 2009.

[10] S. Matsumoto and al. Wide-area situational awareness (wasa) system based upon international standards. In DPSP 2012, pages 1-6, April 2012.

[11] A. G. Phadke and J. S. Thorp. Communication needs for wide area measurement applications. In $C R I S$, pages 1-7, Sept 2010.

[12] K. Budka, J. Deshpande, and M. Thottan. Communication Networks for Smart Grids: Making Smart Grid Real. Springer Publishing Company, Incorporated, 2014. 